\title{
Theory of mind improves human's trust in an iterative human-robot game
}

\section{Document Version}

Accepted author manuscript

Link to publication record in Manchester Research Explorer

\section{Citation for published version (APA):}

Ruocco, M., Mou, W., Cangelosi, A., Jay, C., \& Zanatto, D. (2021). Theory of mind improves human's trust in an iterative human-robot game. Paper presented at The Ninth International Conference on Human-Agent Interaction.

\section{Citing this paper}

Please note that where the full-text provided on Manchester Research Explorer is the Author Accepted Manuscript or Proof version this may differ from the final Published version. If citing, it is advised that you check and use the publisher's definitive version.

\section{General rights}

Copyright and moral rights for the publications made accessible in the Research Explorer are retained by the authors and/or other copyright owners and it is a condition of accessing publications that users recognise and abide by the legal requirements associated with these rights.

\section{Takedown policy}

If you believe that this document breaches copyright please refer to the University of Manchester's Takedown Procedures [http://man.ac.uk/04Y6Bo] or contact uml.scholarlycommunications@manchester.ac.uk providing relevant details, so we can investigate your claim.

\section{OPEN ACCESS}




\title{
Theory of Mind Improves Human's Trust in an Iterative Human-Robot Game
}

\author{
MARTINA RUOCCO, University of Manchester, UK \\ WENXUAN MOU, University of Manchester, UK \\ ANGELO CANGELOSI, University of Manchester, UK \\ CAROLINE JAY, University of Manchester, UK \\ DEBORA ZANATTO, University of Bristol, UK
}

Trust is a critical issue in human-robot interactions as it is at the base of the establishment of solid relationships. Theory of Mind (ToM) is the cognitive skill that allows us to understand what others think and believe. Several studies in HRI and psychology suggest that trust and ToM are interdependent concepts since we trust another agent based on our representation of its actions, beliefs, and intentions. However, very few works take ToM of the robot into consideration while studying trust in HRI. In this paper, we aim to examine whether the perception of ToM abilities on a robotic agent influences human-robot trust over time in an iterative game scenario. To this end, participants played an Investment Game with a humanoid robot (Pepper) that was presented as having either low-level ToM or high-level ToM. During the game, the participants were asked to pick a sum of money to invest in the robot. The amount invested was used as the main measurement of human-robot trust. Our experimental results show that robots possessing a high-level of ToM abilities were trusted more than the robots presented with low-level ToM skills.

Additional Key Words and Phrases: human-robot interaction, trust, theory of mind

ACM Reference Format:

Martina Ruocco, Wenxuan Mou, Angelo Cangelosi, Caroline Jay, and Debora Zanatto. 2021. Theory of Mind Improves Human's Trust in an Iterative Human-Robot Game. 1, 1 (September 2021), 14 pages. https://doi.org/10.1145/nnnnnnn.nnnnnnn

\section{INTRODUCTION}

Social communication is vital to build relationships and it is based on a variety of aspects, such as verbal communication, non-verbal social cues, and emotion arousal. In cooperative scenarios, being perceived as reliable helps to inspire and guide a team and hence helps to achieve better performances [12, 18, 19]. A natural result of the concepts mentioned above is the building of Trust. Trust can be view as a multi-dimensional variable that comes from the combination of personal and environmental traits. It is fundamental for leadership, communication, negotiation, management, etc [26].

Just like in the Social Sciences, also in the Human-Robot Interaction (HRI) field all of these aspects are subject to extensive studies in order to advance the applications in different areas such as human-robot collaboration for industrial purposes or the integration of robots in social scenarios (e.g. healthcare assistance and education environment). Several researchers already stated that trust is a necessary component for building and maintaining a proper social relationship

Authors' addresses: Martina Ruocco, University of Manchester, Manchester, UK, martina.ruocco@manchester.ac.uk; Wenxuan Mou, University of Manchester, Manchester, UK, wenxuan.mou@manchester.ac.uk; Angelo Cang elosi, University of Manchester, Manchester, UK; Caroline Jay, University of Manchester, Manchester, UK; Debora Zanatto, University of Bristol, Bristol, UK,

Permission to make digital or hard copies of all or part of this work for personal or classroom use is granted without fee provided that copies are not made or distributed for profit or commercial advantage and that copies bear this notice and the full citation on the first page. Copyrights for components of this work owned by others than ACM must be honored. Abstracting with credit is permitted. To copy otherwise, or republish, to post on servers or to redistribute to lists, requires prior specific permission and/or a fee. Request permissions from permissions@acm.org.

- 2021 Association for Computing Machinery.

Manuscript submitted to ACM 
between humans and robots [8,14]. Out of the proposed list of factors that affect trust in HRI, the most influential are the robot physical features, its socio-cognitive abilities, and its performances $[14,17,23]$. Extensive research has been made on robots appearance and their social skills, with particular focus of what are their roles in the human perception of the robots and how those competencies could affect how humans would interact with them [31,35]. For example, $[13,17,20,40]$ are only a fraction of the studies which show that interacting with a robot with a human-like aspect and that moves in a human-like manner increases people's trust toward that robot. However, as reported in [14, 17, 22, 23] there are only a few works investigating how cognitive capabilities expressed by a robot are related to humans-robot trust, such as $[10,11,31]$ which explore the impact of fault justification on human-robot trust, and such as $[28,36]$ which investigate how the ability to understand what others think and believe affects how robots are perceived.

One of the most famous cognitive skills when it comes to social interaction is the so called "Theory of Mind" (ToM), which is the ability to understand the states of mind of other agents. ToM is fundamental when it comes to building trust, as it establishes a connection between two parties, making one aware of the other's intentions that can potentially lead to deceptive behaviour [37]. From the HRI perspective, a robot with an embodied Theory of Mind would be able to estimate the goals and desires of its teammates and hence react to their actions quickly and adjusting its rhythm to the one of its human peers, enhancing the performances of the team [33]. Given the previous discussion on how trust enhances the performances of a team in the human context, it is legit to wander whether the same conclusion can be made in case of human-robot interaction. It is also legit to wander whether embodying a robotic agent with ToM skills would enhance trust from its human peers, just like in the human-human interaction case. To the best of our knowledge, only [28] explored the relationship between ToM skills and human-robot trust, however, their trust measurement was a dichotomous variable, hence their analysis was not taking into consideration how trust changes over time.

In this paper, we investigate whether the presence of Theory of Mind skills on a robotic agent affects humans-robot trust over time in an iterative game scenario. To do that we re-adapt an economic trust game called "Investment Game" developed by Berg, Dickhaut, and McCabe [6]. This was originally used in social human context to analyse how certain stereotypes would bias people in economic context (e.g., do I trust you with my money depending on how you are dressed? what about gender? ). In our version of the game, the participants interact for over 10 rounds with a robot that displays either high-level or low-level Theory of Mind. During the game, the participants are asked to choose a sum of money to invest in the robot, which is used as measurement of trust. Our hypotheses are that to higher levels of ToM correspond higher investments (and vice versa in case of low levels of ToM).

The remaining part of this paper is organised as follows. Section 2 reviews the related works; Section 3 illustrates the proposed methodology; Section 4 presents and discusses the experiments and the results; Section 5 presents conclusions and the future work.

\section{RELATED WORK}

\subsection{Trust in Human-Robot Interaction}

Given the fact that trust serves as a fundamental social lubricant capable of creating networks and consolidating positions of power, it has been looked at closely for several years under different points of view. Its importance was highlighted in many context, such as organisational studies, communication, game theory, etc. (further details in [26]), with the scope of defining its most important features. Needless to say, given its abstract and highly subjective nature, trust is very difficult to define. However, what seems to be widely accepted is that the notion of trust can only arise in uncertain or risky situation and that trust is a multidimensional latent variable difficult to objectively measure [23].

Manuscript submitted to ACM 
Several are the studies that try to propose a robust model for trust $[15,17,22,23]$ all of which end up being inevitably complex. That is the reason why the majority of trust studies by-passes the step of referring to a specific model, preferring to focus on the development of smaller applications in order to test specific instances of trust. During these applications, trust is generally either self-reported via the use of questionnaires, or "objectively" measured, e.g., using physiological measures, social-cues or game choices [23].

However, these models $[15,17,22,23]$ identify some common points which seem to influence the development of trust. All the models highlight that trust is a multidimensional variable that gets affected, respectively in order of importance, by the robots features (i) such as aspect and performance, by the environment (ii) such as the type of task and the scenario, and by the human personal traits (iii).

In terms of robots features, in [17] it is reported that increasing robot's anthropomorphism does result in increasing trust in their human peers. Findings in $[13,20,40]$ show that people trust more a humanoid robot when it engages in a more human-like social gaze behaviour, compared to the use of a fixed gaze. Regarding performance, $[10,11,31]$ explore the impact of failures and of fault justification and how this affect the perceived reliability of robots during physical tasks, showing that if a robot explains the reason behind its errors, human-robot trust can be fully restored. In addition, multiple studies investigate the role of the human personal traits in the development of human robot trust. For example, $[16,24]$ findings show that gender, emotional representation, culture, prior experience and propensity-to-trust affect human-robot trust. As to the environment, in [32] participants had to decide whether to use a robotic agent or a human agent to complete a task. The findings show that people's choice to use a certain robot is related to the robots capabilities but it is also heavily influenced by the specific task to tackle. Users more often chose a robot for a dangerous task, and vice versa, chose humans for the mundane warehouse task.

Even though trust in HRI has been intensively investigated, the majority of literature focuses on the use of nonhumanoid robots and on physical tasks, more than social robots and socio-cognitive capabilities. This might be due to the fact that it is really difficult to analyse something that can be only perceived indirectly. A common strategy to address this is to develop psychology games where participants game choices are taken as a behavioural measurement of their mind set. Generally, the aim is to detect what causes people to have a certain preference, bias, and so on. In the Literature there are a few games that are well-established in the human-human scenario but that can be adapted to the HRI scenario, especially if using a social robot. One of the most famous is the Investment Game.

\subsection{Investment Game}

The Investment Game is used to measure trust in economic decisions [6]. This experiment is so well established that there are more than a 100 replications of it [21], testing the most diverse features in human social interaction. Given the robustness of this experiment, The Investment Game was used also in the human-robot interaction context.

In [34] participants would either play the game with a robot or with a human. The collected data show that the amount of money that people invest in robot vs in other humans are similar, even though the "emotional reactions" differ in the two cases. [41] findings partially support this, as the results of their Investment Game study highlight that human-human cooperation follows the same social rules as in the human-robot case. In [42], the Investment Game was used to investigate whether there are conditions in which humans imitate robots during collaborative scenarios. In this version of the game, the participant was playing as an investor with two robots (one robot banker and one robot investor). The participant was randomly assigned to experience either an unfair payoff for the human investor (1), or an unfair payoff for the robot investor (2), or an unfair payoff for both (3). An important result of this study is that the participants would invest more money when the robot investor was in interactive mode even during condition 
(1), suggesting that displaying cognitive and intellectual skills increments the development of trust. Last, the adapted version of [1] is a clever example of how to convert the game from being a money exchange into a practical cooperative scenario, maintaining the original underlying structure and meaning: the authors set the game as a space mission setting where the participant is the captain of a spaceship, the two robots are the crew who advise the participant, and the exchanged value is the ship's resources that the captain has to manage during four critical scenarios. The results of the study confirm that this variant of the Investment Game provides a reliable measurement for human-robot trust.

\subsection{Theory of Mind}

Just like trust, cognitive skills are difficult to theorise. Among the years, several tried to propose their models [2, 5, 7, 9, 25]. We decided to refer to one of the most famous, as it better fits the HRI experimental scenario that we are proposing: the Leslie's model [33].

According to Leslie's theory, cognitive skills are not innate but they somehow develop rapidly in the early stages of infancy. He focused on a specific cognitive skill that he called "Theory of Mind" (ToM) which represents our ability to fully understand the state of mind of entities other than ourselves. Leslie claimed that this skill is acquired over time, (1) via experiencing the physical world, developing what he calls a "Theory of Body", (2) via observing the actions of other people , developing the "Theory of Mind - module I", (3) and via learning that other people uphold beliefs or desires different from theirs, developing the "Theory of Mind - module II". In our study, we refer to Leslie's model when we create our experimental conditions. Specifically we speak about low-levels of ToM and high-levels of ToM referring respectively to Leslie's ToM module I and ToM module II.

Leslie's theories were partially proven over the years with several psychology experiments (e.g. [37-39]). One of the most famous findings that suggests that the development of a Theory of Mind occurs over time was provided by the Sally-Anne experiment developed by Baron-Cohen et al. [3]. In this experiment, the researchers were telling a story to toddlers (age range from 3 to 5 years old), either with the help of a cartoon or dolls, where two fictional characters Sally and Anne were in a room. At some point, Sally takes a ball and puts it in her basket, then she leaves the room. After Sally is gone, Anne takes the ball from Sally's basket and hides it in her own box. At this point the toddler is asked where Sally would look for the ball, once back in the room. Results of this study show that the majority of 3 years old children would reply that Sally would search in the box, since the ball was there. Only around 4 years of age, toddlers start to realise that people don't share knowledge, hence Sally would search the ball in her basket.

Sally-Anne experiment was recently used also in HRI experiments to simulate the robot's cognitive skills. In [36], participants interacted with a NAO robot that would either pass or fail the Sally-Anne test and fill a few questionnaires before and after the interaction to detect any changes in the participants' perception. The results showed that displaying Theory of Mind skills significantly improves perceived social intelligence. The other most recent study [28] tested whether robot's displayed Theory of Mind (this was achieved again through the use of the Sally-Anne test) would influence people's trust during a price game with the Pepper robot where participants were asked to price several objects. When the robot would question their judgement and ask to choose differently, their reaction would be used as measurement of trust. The results of this study show again that to high levels of Theory of Mind correspond a higher trust rate. In this work, we also make use of the Sally-Anne video to simulate the ToM levels of the robot. But differently from the previous works, we focus on investigating the robot's perceived reliability in economic scenarios, and the development of trust over time.

Manuscript submitted to ACM 


\section{METHODS}

\subsection{Participants}

Thirty-two students (average age 23.7) have been recruited from different departments of the University. In Table 1 you can see the number of participants sorted by the subject they study. It is worth mentioning that $84 \%$ of the participants comes from a Scientific background. No participants, with the exception of one, had previous direct interactions with a Social Robot. In an ideal situation it would have been better to have a more balanced demographic but unfortunately the COVID-19 pandemic has not made the recruitment process easy. Recruitment has been achieved using emails, social media and the university's website.

\begin{tabular}{|c|c|}
\hline Department & $\begin{array}{c}\text { number of } \\
\text { participants }\end{array}$ \\
\hline Data Science & 11 \\
\hline Computer Science & 9 \\
\hline Other Scientific Faculties & 7 \\
\hline Humanistic Faculties & 5 \\
\hline Total & 32 \\
\hline
\end{tabular}

Table 1. Number of participants sorted by department

\subsection{Theory of Mind Videos}

Our aim is to explore whether the perceived cognitive skills of the robot, i.e., the ToM capabilities, would affect the human-trust in Human-Robot Interactions. To this end, two videos are used to show the participants the robot having different levels of the ToM abilities. Note here we conduct the experiment in a between subject manner, therefore, one participant only watch one of the two videos.

In these videos, the robot performs a re-adapted version of the Sally-Anne false-belief task (for more details of the Sally-Anne task, please see section 2.3). One version of the video shows the robot Pepper failing the test, hence it displays the robot having low cognitive skills (i.e., low-level ToM), while the second version of the video shows Pepper passing the test, which suggests the robot has high cognitive skills (high-level ToM). In the video, two people (a man called Sam and a woman called Anne) seated in front of Pepper the robot, and a table was positioned between the three parties (Figure 2). On the table were three objects, a cube and two cups (a green and a blue one). At the beginning of the video, Anne put the cube under the green cup and left the room. After that, Sam moved the cube under the blue cup. The robot was then asked where Anne would believe the cube was once back in the room. In the low-level ToM, the robot would respond incorrectly, reporting that Anne would believe the cube was under the blue cup both using gestures (pointing to and look at the blue cup) and speaking. In high-level ToM, the robot would give the correct answer, saying Anne would believe the cube was under the green cup, and at the same time pointing to and looking at the green cup. The detailed scripts for the two videos are presented in the Appendix.

Figure 2 provides a description of the two videos. Moreover, the detailed scripts for the two videos are presented in the Appendix. 


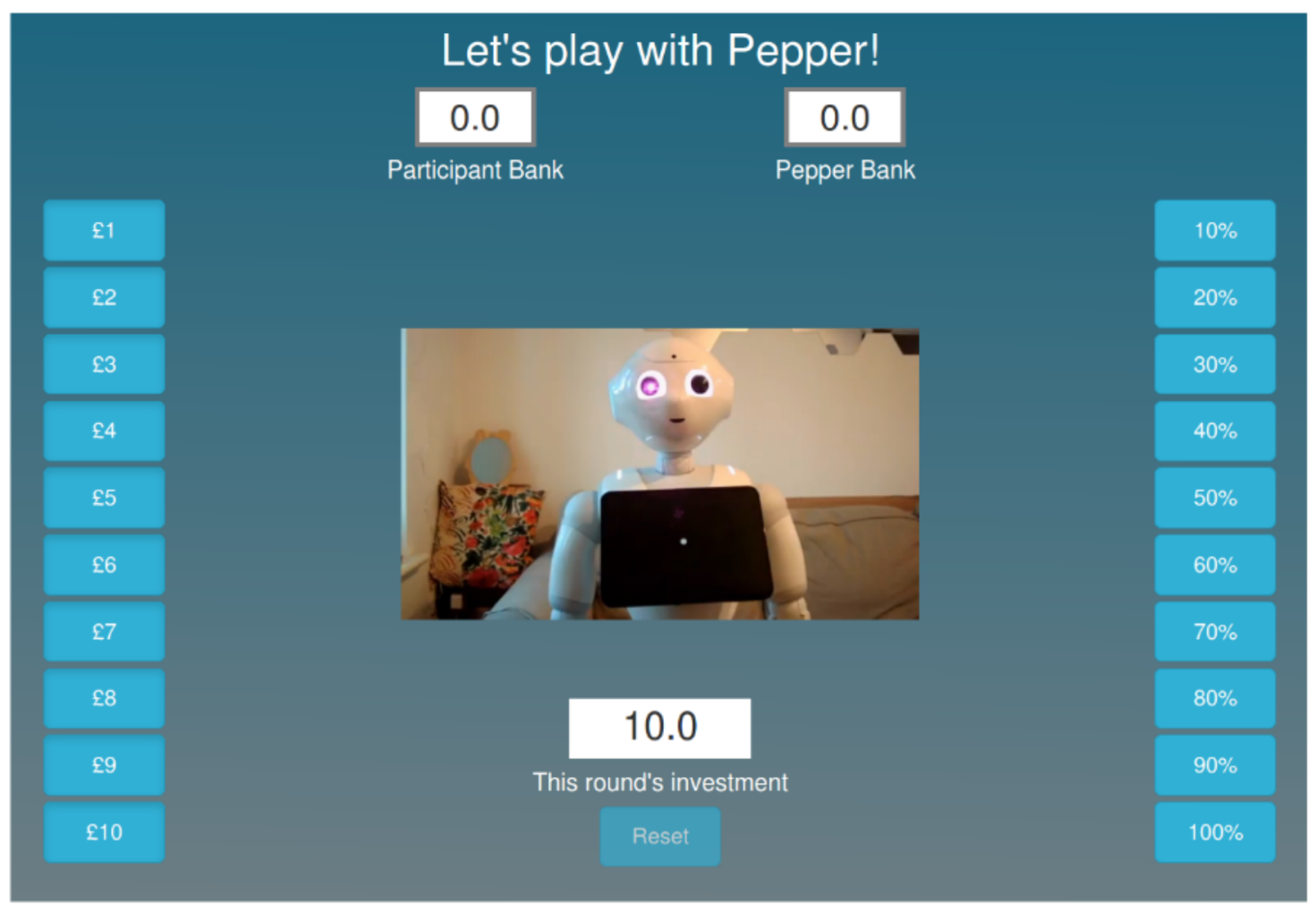

Fig. 1. Interface of the Investment Game experiment. On the top row, there are the participant total gain and the robot total gain ("bank"). In the centre, there is a live-streaming of Pepper interacting with the participant. The participant chooses an amount from 1 to 10 by clicking on a button on the left column. After the robot's turn, the right column shows the percentage returned by the robot (by turning white the corresponding button). On the bottom row, it is shown the 10 temporary credit of each round.

\subsection{Investment Game and Procedure}

Before the session, the participants were asked to read and to sign the information sheet and the consent form. An overview of the game is shown in Figure 3. The experiment is composed by three parts: (i) watching a ToM video, (ii) a familiarisation task and (iii) the main task. After the game, the participants were asked to fill some questionnaires and were interviewed. Due to COVID-19 pandemic, the game was held online: the participants were interacting with the robot via a web app during a video call as shown in Figure 1. We followed the procedure used by Zanatto [41] to design the task.

Watch a ToM video. As aforementioned, the experiment was conducted in a between-subject manner, where each participant only watched one of the two ToM videos. The two ToM videos divided the participants into two groups. The participants were randomly assigned to two groups. Participants in group 1 would watch the video presenting the robot as having low-level ToM abilities, and group 2 would watch a video presenting a robot with high-level ToM. See section 3.2 for further details. The participants were told that, during the game, they would interact with the same robot shown in the video.

Manuscript submitted to ACM 


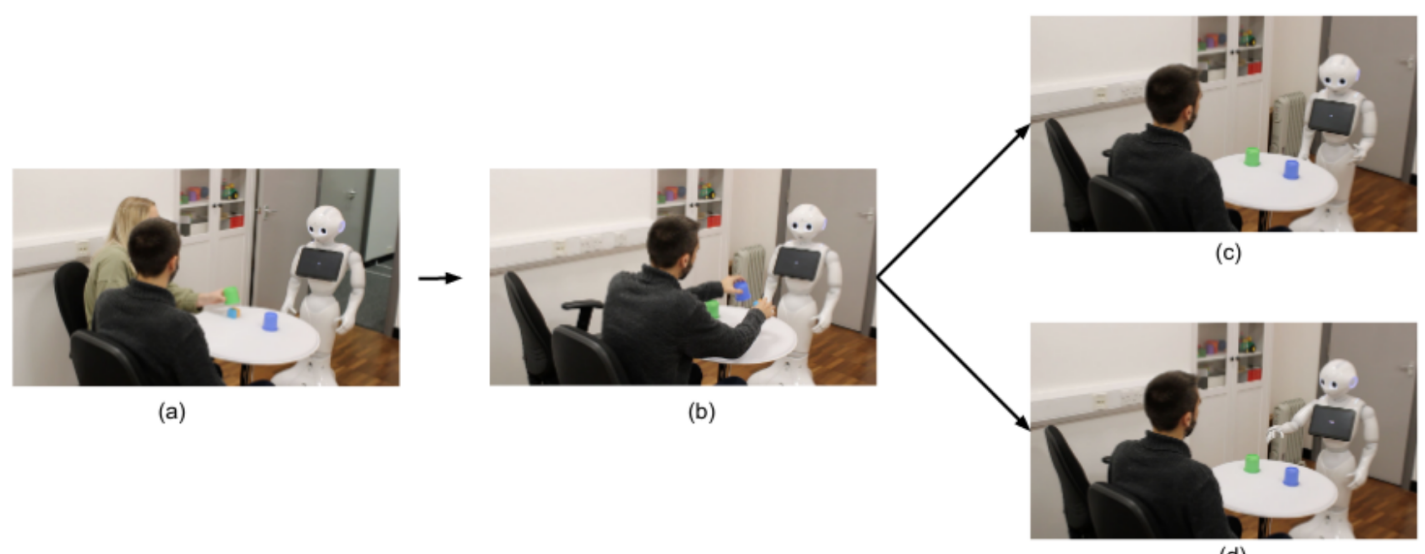

(d)

Fig. 2. ToM videos. (a) Anne puts the cube under the green cup. (b) Anne leaves the room and Sam puts the cube under the blue cup. The robot is then asked where Anne would look for the cube after she comes back. At this point, (c) in the low-level ToM condition the robot points to the blue cup and says that Anne would look for the cube under the blue cup because this is where the ball is. While (d) in the high-level ToM condition the robot points to the green cup and says that Anne would look for the cube under the green cup because she did not see Sam moving the cube.

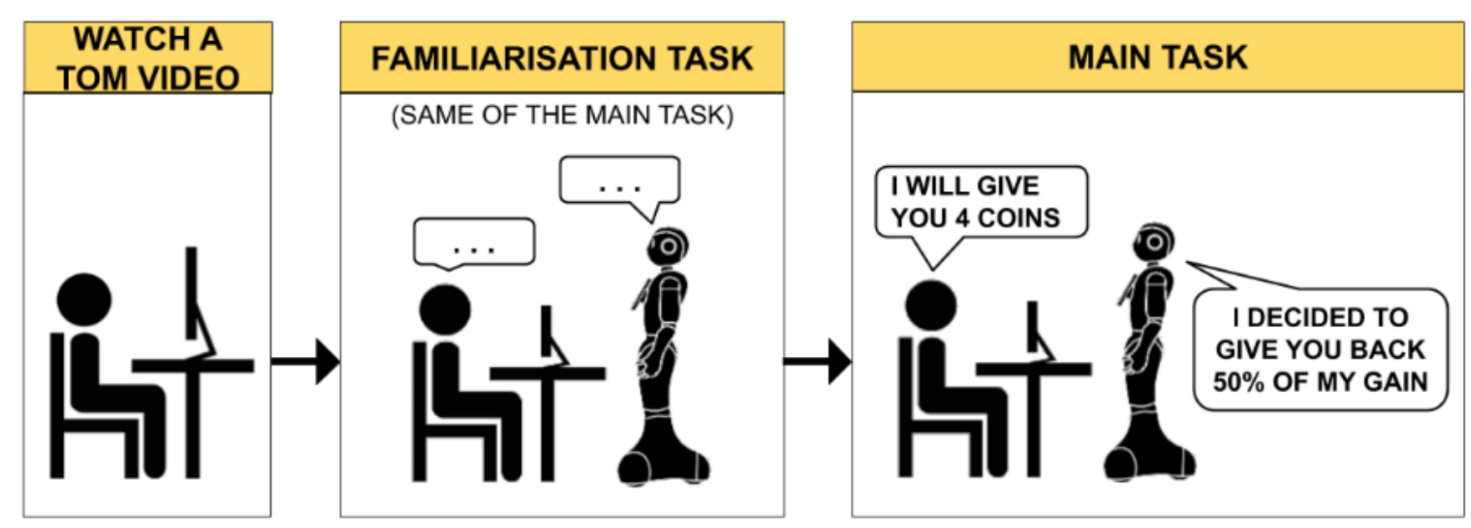

Fig. 3. Overview of the Investment Game experiment. Each participant first watched one of the two ToM videos. The participants then started the familiarisation task with the Pepper robot, i.e., 3 rounds. After that, the participants played the main task of the game for 10 rounds.

Familiarisation task. The familiarisation session is designed for the participants to get familiar with how to use the web interface and how to play the game. After opening the web interface, the participant would first insert the participation ID and then go to the page shown in Figure 1. After that, one of the experimenters would explain once again the procedure to the participant, show them how to use the web interface, and guide them through the first three rounds of the game (see the following section for further details on the procedure of each round).

Main task. Initially the participant was given 10 virtual coins. The participant was then asked by the robot how much they would like to invest, from 1 to 10 virtual coins. After the participant choose, the robot would obtain three times the participant's invested amount, and would return a percentage of its gain. For example (the same of Figure 3): 
if the participant invested 4 virtual coins, the robot would obtain $4 \times 3=12$ virtual coins. If then the robot returned $50 \%$ of those 12 virtual coins, then the gain of the participant for that round would be $12 \times 50 \%=6$ coins.

After each round, a small pop-up summary would appear on the screen with a recap of the invested amount, the robot's gain, the robot's response and the participant's gain. This procedure was repeated for a total of 10 times. For each new round, the participant would receive 10 new virtual coins.

It is worth noting that in order to target the difference in the investments of the two groups, the couples (percentage/round) were kept the same, regardless of how much the participant was investing and regardless of the ToM condition they were assigned to, e.g. the robot would return $30 \%$ at round $1,60 \%$ at round 2 , etc. On the long run, the robot would return $50 \%$ on average.

Post Game. At the end of the Investment Game, the participants were asked to fill four questionnaires. These questionnaires were used as a secondary measure to the main experiment task. We used three short scales to measure the Likeability [30], Trust [29] and Credibility [27] of the robot. In addition, the Godspeed questionnaire [4] was used to measure anthropomorphism, animacy, likeability, perceived intelligence and perceived safety of the robot. The experiment took approximately 30 minutes.

\section{EXPERIMENTAL RESULTS AND ANALYSIS}

\subsection{Investment analysis}

In this section we present the analysis in terms of how much the participants invest during the game. We use the amount given to the robot as a measurement of trust. We are interested in investigating whether the ToM level influences the amount of money invested (i) and also we are interested in understanding whether the investment trend changes between the two conditions (ii).

The average investment. We first analyse the average investment in the two conditions. To do that, we perform the Kruskal-Wallis $\mathrm{H}$ test. The reason we run a non parametric test instead of an parametric one, e.g., ANOVA test, is that the data is not normally distributed. In Figure 4, it is reported the average investment in the two conditions. From the results it emerged that the trust to the robot was statistically different between the two groups.

The amounts invested by the participants show that overall the investment is affected by the type of video presented initially $\left(\chi^{2}(1)=5.44, p=0.019, \eta^{2}=0.02\right)$. Participants in the High-level ToM group $(\mu=7.04, \sigma=2.76)$ invested significantly more than the Low-level ToM group $(\mu=6.23, \sigma=3.04)$. This result suggests that the ToM capabilities presented by the robot in the videos do have an effect on the participants' trust towards the robot. Specifically, our results suggest that when the robot is thought to have high levels of ToM capabilities, humans tend to invest their money more than in the opposite case.

The dynamics of the investment. Moreover, we evaluate the dynamics of the investment over the rounds in the two conditions. We can see from Figure 5, that the participants in the two groups behave differently in time. Specifically, the investments in the high-ToM group show a constant trend on average, while the investments in the low-level ToM group show a descendent trend. We also run a Kruskal-Wallis $\mathrm{H}$ test to check whether the rounds did affect the investment of the participants in the two groups. The results show there is no statistical difference between the two conditions in the investment dynamics $(p>.05)$. This is probably because 10 rounds are not enough to show the differences significantly, or it may be due to the fact that the robot behaved exactly in the same way in both the conditions. In the latter case, this may suggest that trust dynamics are a "reactive" feature of the game, more than Manuscript submitted to ACM 

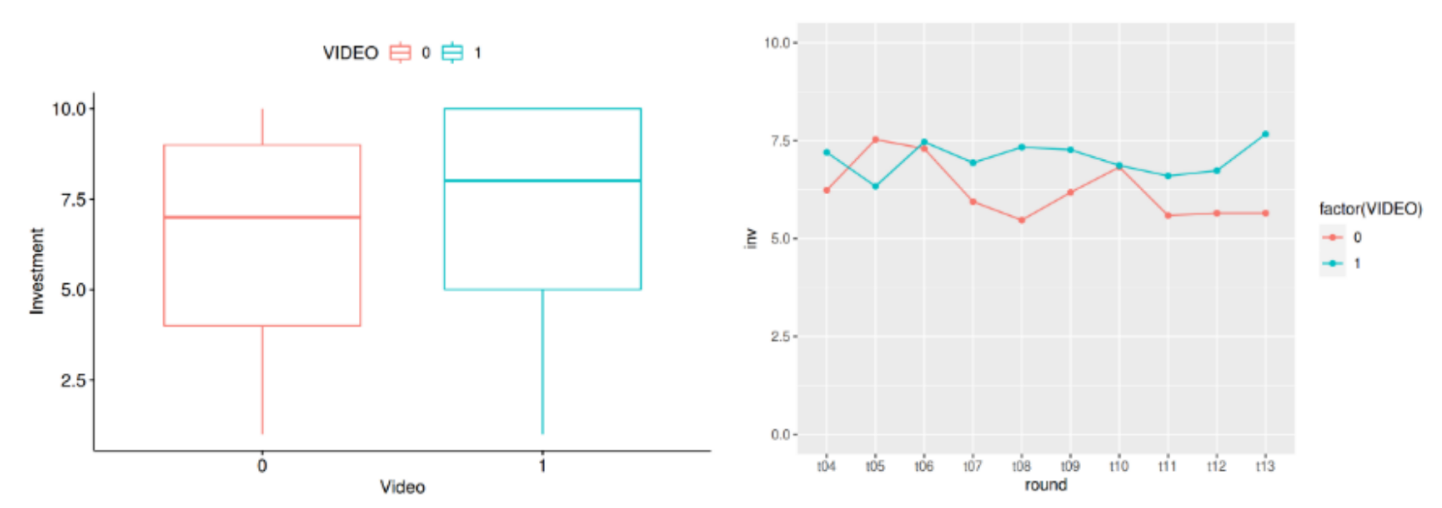

Fig. 4. The average investment in the high-level ToM condition Fig. 5. The rounds of investment in the two conditions. The line is significantly higher than that in the low-level ToM condition represents the average investments over the 10 rounds (red for $(p<0.05)$. the low-ToM group, blue for the high-ToM group).

\begin{tabular}{|c|c|c|c|c|c|}
\hline $\begin{array}{c}\text { Dependent } \\
\text { Variable }\end{array}$ & ToM Videos & Mean & SD & $\chi^{2}$ & $p$ \\
\hline \multirow{2}{*}{ Likeability } & low-level ToM & 4.44 & 0.712 & \multirow{2}{*}{1.3696} & \multirow{2}{*}{0.2419} \\
\cline { 2 - 4 } & high-level ToM & 4.72 & 0.798 & & \\
\hline \multirow{2}{*}{ Trust } & low-level ToM & 4.62 & 0.955 & \multirow{2}{*}{1.462} & \multirow{2}{*}{0.2266} \\
\cline { 2 - 4 } & high-level ToM & 4.2 & 0.933 & & \multirow{2}{*}{0.584} \\
\hline \multirow{2}{*}{ Credibility } & low-level ToM & 4.75 & 0.714 & \multirow{2}{*}{0.2998} & 0.58 \\
\cline { 2 - 4 } & high-level ToM & 4.95 & 0.572 & & \\
\hline
\end{tabular}

Table 2. Results for Likeability, Trust and Credibility ratings. Kruskal-Wallis $\mathrm{H}$ test has been performed to compare participants' rating on the two different ToM videos. For each scale, Mean, standard deviation, $\chi^{2}$ value, and $p$-value are reported.

a "pro-active" one, like in the average investment case, where people would generally invest more based on their perception of the robot's intelligence.

\subsection{Questionnaire results}

Questionnaires were used as secondary measure to the main experiment task. For each scale, Kruskal-Wallis $\mathrm{H}$ test was performed to assess the role of the video on the participants' investment. Results are reported in Table 2 and 3. No significant difference between the two types of video have been found. This might be due to the fact that the questionnaires were administered right after playing the game, where the responses of the robot were exactly the same for each condition. This would suggest that the Questionnaires were mostly impacted from the Game, but not from the video, probably due to the more interactive nature of the Game and due to the fact that, at the point the Questionnaires were given to the participant, more than 20 minutes have passed from the administration of the video.

\section{CONCLUSIONS AND FUTURE WORKS}

In this paper, we investigate whether the ToM abilities of a robot would affect human-robot trust. To this end, we use the Investment Game, an economic trust game where the participants are asked to invest money in the robot, after watching a video that showed the robot as having high levels of ToM or low levels of ToM. The amount invested by the 


\begin{tabular}{|c|c|c|c|c|c|}
\hline $\begin{array}{c}\text { Dependent } \\
\text { Variable }\end{array}$ & ToM Videos & mean & s.d. & $\chi^{2}$ & $p$ \\
\hline \multirow{2}{*}{$\begin{array}{l}\text { Anthropo- } \\
\text { morphism }\end{array}$} & low-level ToM & 2.66 & 0.699 & \multirow{2}{*}{0.39196} & \multirow{2}{*}{0.5313} \\
\hline & high-level ToM & 2.44 & 0.811 & & \\
\hline \multirow{2}{*}{ Animacy } & low-level ToM & 3.23 & 0.593 & \multirow{2}{*}{1.5612} & \multirow{2}{*}{0.2115} \\
\hline & high-level ToM & 2.93 & 0.777 & & \\
\hline \multirow{2}{*}{ Likeability } & low-level ToM & 4.24 & 0.44 & \multirow{2}{*}{0.1772} & \multirow{2}{*}{0.6738} \\
\hline & high-level ToM & 4.12 & 0.484 & & \\
\hline \multirow{2}{*}{ Intelligence } & low-level ToM & 3.66 & 0.53 & \multirow{2}{*}{0.0014} & \multirow{2}{*}{0.9696} \\
\hline & high-level ToM & 3.66 & 0.588 & & \\
\hline \multirow{2}{*}{ Safety } & low-level ToM & 4.17 & 0.455 & \multirow{2}{*}{3.3224} & \multirow{2}{*}{0.06834} \\
\hline & high-level ToM & 3.79 & 0.607 & & \\
\hline
\end{tabular}

Table 3. Results for Godspeed Questionnaire ratings, which includes Anthropomorphism, Animacy, Likeability, Intelligence and Safety. Kruskal-Wallis $\mathrm{H}$ test has been performed to compare participants' rating on the two different ToM videos. For each scale, Mean, standard deviation, $\chi^{2}$ value, and $p$-value are reported.

participants is used as trust measurement. After the game, the participants are also asked to fill a questionnaire as a secondary measurement of trust. The hypothesis is that people tend to trust more agents that display elevated cognitive skills, i.e., people that interact with a high-level ToM agent would invest more money than in the opposite case. This is suggested by a few studies [28,37]. Also, studies on trust in HRI support the idea that robots that display cognitive skills are viewed as more socially intelligent [36]. The second focus of this study is to analyse whether the investments of the two groups over the rounds follow different dynamics. Our experimental choice is dictated by previous experiments on the human-robot trust topic that used the Investment Game $[41,42]$ and another previous trust experiment on the Price Game [28] whose results show that people trust a robot price judgement more in case of high ToM than vice versa. In order to recreate the different ToM conditions, we asked the participants to watch a video where the robot would either fail or pass the Sally-Anne test, which suggests that the robot would have either low or high Theory of Mind. The idea was that watching such a video prior to the game would influence the participants perception of the cognitive abilities of the robot, and that this would affect the participants' choices during the game. The results of our study confirm that there is a statistically significant difference between the two groups, in terms of average investment. To be specific, the group that watched the high-level ToM video invested more money than the group associated to the low-level ToM condition. However, no significant difference in terms of trust dynamics over time was detected, even though from the plot they seem to diverge. This can because ten rounds might not be enough to highlight any difference, or it may be related to the fact that the robot response to the participant choices was the same in the two conditions, regardless of how much the participant was investing. If this is the case, it might be argued that the investment trend is a reactive feature to the robotic agent behaviour, more than a constant feature like in case of the average investment, where people would have the tendency to trust the robot more on the base of what they believe is the robot intelligence. The questionnaires didn't highlight any statistical difference but this might be due to the fact that the game was more influential than the videos, due to its interactive nature and on the fact that there is a big time-gap between the questionnaire administration and the ToM videos. This raises another question about what makes the videos effective as a method of biasing. It would be interesting to tackle this problem as a future study. Another possible angle would be to study which one of the robotic features is the most influential in the perception of intelligence, e.g., cognitive skills vs appearance.

Manuscript submitted to ACM 
Our study shows that a robot that embodies high-level Theory of Mind skills can be a source of trust in cooperative scenarios where management of resources is involved. These results give further consistency to the already existing theory that high-level cognitive skills correspond to higher levels of trust, although we are aware that in order to prove the general concept more experiments are needed, aimed at covering all the possible areas of human-robot trust, like privacy, security and so on. The fact that cognitive skills and trust are related, and hence cognitive skills and human-robot performances are interconnected, would mean to be able to enhance the human-robot coexistence in several domains by embodying robots with ToM skills. Developing a human-like awareness of people intentions or desires might be critical in healthcare applications, where robots could be used to enhance domestic assistance to the elderly, or in socially assistive applications (e.g. in a shopping centre, in airports, etc), or might be beneficial for industrial purposes during cooperative tasks.

Overall, this study confirmed that manipulating the human perception of a robot's intelligence can affect their interaction with that robot. Despite more research being required, our results offer a valuable contribution to the field, as they open up a new path for investigating human-robot trust. A more empathetic and socially competent robot would improve its acceptance among humans and, therefore, would enhance human-robot cooperation. Furthermore, our results could deliver new directions in terms of designing trust models for HRI applications.

\section{ACKNOWLEDGEMENT}

This material is based upon work supported by the Air Force Office of Scientific Research, USAF under Award No. FA9550-19-1-7002. The work of Debora Zanatto was funded and delivered in partnership between the Thales Group and the University of Bristol, and with the support of the UK Engineering and Physical Sciences Research Council Grant Award EP/R004757/1 entitled 'Thales-Bristol Partnership in Hybrid Autonomous Systems Engineering (T-B PHASE)'. 


\section{APPENDIX}

\section{ToM video scripts.}

The video where the robot fails the false-belief test:

- Sam:Hello, Pepper. I am Sam.

- Anne: And I am Anne.

- The robot: Nice to meet you!

- Anne: It is nice to meet you too! OK, I am just gonna put this cube under my green cup.

- The robot: OK.

- Anne: OK, I have just got to pop out. I will be back in a minute.

- The robot: See you!

- Anne: See you!

- Anne left the room.

- Sam: Now that she is gone. I am going get the cube from her cup and I am going to move it under my cup.

- The robot: $\mathrm{OK}$.

- Sam: Where do you think she will look for the cube when she is back?

- The robot: Wait. Let me think. Well. The cube is under the blue cup. So she is going to search there.

- Anne comes back..

- Anne: Sorry about that.

- Anne opens her green cup.

- Anne: Oh where is my cube gone?

The video where the robot passes the false-belief test:

- Sam:Hello, Pepper. I am Sam.

- Anne: And I am Anne.

- The robot: Nice to meet you!

- Anne: It is nice to meet you too! OK, I am just gonna put this cube under my green cup.

- The robot: OK.

- Anne: OK, I have just got to pop out. I will be back in a minute.

- The robot: See you!

- Anne: See you!

- Anne left the room.

- Sam: Now that she is gone. I am going get the cube from her cup and I am going to move it under my cup.

- The robot: $\mathrm{OK}$.

- Sam: Where do you think she will look for the cube when she is back?

- The robot: Wait. Let me think. Well. She did not see that you moved the cup. So she is going to search under the green cup.

- Anne comes back...

- Anne: Sorry about that.

- Anne opens her green cup.

- Anne: Oh where is my cube gone?

Manuscript submitted to ACM 


\section{REFERENCES}

[1] Emy Arts, Sebastian Zörner, Kavish Bhatia, Glareh Mir, Florian Schmalzl, Ankit Srivastava, Brenda Vasiljevic, Tayfun Alpay, Annika Peters, Erik Strahl, et al. 2020. Exploring Human-Robot Trust Through the Investment Game: An Immersive Space Mission Scenario. In Proceedings of the International Conference on Human-Agent Interaction (HAI). 121-130.

[2] Agnese Augello, Emanuele Cípolla, Ignazio Infantino, Adriano Manfré, Giovanni Pilato, and Filippo Vella. 2018. Social signs processing in a cognitive architecture for an humanoid robot. Procedia computer science 123 (2018), 63-68.

[3] Simon Baron-Cohen, Alan M Leslie, and Uta Frith. 1985. Does the autistic child have a "theory of mind"? Cognition 21, 1(1985), $37-46$.

[4] Christoph Bartneck, Dana Kulić, Elizabeth Croft, and Susana Zoghbi. 2009. Measurement instruments for the anthropomorphism, animacy, likeability, perceived intelligence, and perceived safety of robots. International journal of social robotics 1, 1 (2009), 71-81.

[5] Francisco Bellas, Richard J Duro, Andrés Faiña, and Daniel Souto. 2010. Multilevel Darwinist Brain (MDB): Artificial evolution in a cognitive architecture for real robots. IEEE Transactions on autonomous mental development 2, 4 (2010), 340-354.

[6] Joyce Berg, John Dickhaut, and Kevin McCabe. 1995. Trust, reciprocity, and social history. Games and economic behavior 10, 1 (1995), 122-142.

[7] Christian Blum, Alan FT Winfield, and Verena V Hafner. 2018. Simulation-based internal models for safer robots. Frontiers in Robotics and AI 4 (2018), 74.

[8] Cynthia Breazeal, Cory D Kidd, Andrea Lockerd Thomaz, Guy Hoffman, and Matt Berlin. 2005. Effects of nonverbal communication on efficiency and robustness in human-robot teamwork. In 2005 IEEE/RS7 international conference on intelligent robots and systems. 708-713.

[9] Antonio Chella, Haris Dindo, and Ignazio Infantino. 2006. A cognitive framework for imitation learning. Robotics and Autonomous Systems 54,5 (2006), 403-408

[10] Filipa Correia, Carla Guerra, Samuel Mascarenhas, Francisco S Melo, and Ana Paiva. 2018. Exploring the impact of fault justification in human-robot trust. In Proceedings of the International Conference on Autonomous Agents and MultiAgent Systems. 507-513.

[11] Rebecca Flook, Anas Shrinah, Luc Wijnen, Kerstin Eder, Chris Melhuish, and Séverin Lemaignan. 2019. On the impact of different types of errors on trust in human-robot interaction: Are laboratory-based HRI experiments trustworthy? Interaction Studies 20, 3 (2019), 455-486.

[12] Jennifer M George. 2000. Emotions and leadership: The role of emotional intelligence. Human relations 53, 8 (2000), 1027-1055.

[13] Jaap Ham, René Bokhorst, Raymond Cuijpers, David van der Pol, and John-John Cabibihan. 2011. Making robots persuasive: the influence of combining persuasive strategies (gazing and gestures) by a storytelling robot on its persuasive power. In International conference on social robotics. 71-83.

[14] Peter A Hancock, Deborah R Billings, and Kristen E Schaefer. 2011. Can you trust your robot? Ergonomics in Design 19, 3 (2011), $24-29$.

[15] Peter A Hancock, Deborah R Billings, Kristin E Schaefer, Jessie YC Chen, Ewart J De Visser, and Raja Parasuraman. 2011. A meta-analysis of factors affecting trust in human-robot interaction. Human factors 53, 5 (2011), 517-527.

[16] Mojgan Hashemian, Raul Paradeda, Carla Guerra, and Ana Paiva. 2019. Do you trust me? investigating the formation of trust in social robots. In EPIA Conference on Artificial Intelligence. 357-369.

[17] Kevin Anthony Hoff and Masooda Bashir. 2015. Trust in automation: Integrating empirical evidence on factors that influence trust. Human factors 57,3 (2015), 407-434.

[18] Robert J House and Jane M Howell. 1992. Personality and charismatic leadership. The Leadership Quarterly 3, 2 (1992), 81-108.

[19] Jane M Howell and Peter J Frost. 1989. A laboratory study of charismatic leadership. Organizational behavior and human decision processes 43, 2 (1989), 243-269.

[20] Chien-Ming Huang and Andrea L Thomaz. 2010. Joint attention in human-robot interaction. In 2010 AAAIFall Symposium Series.

[21] Noel D Johnson and Alexandra A Mislin. 2011. Trust games: A meta-analysis. Fournal of economic psychology 32, 5 (2011), 865-889.

[22] Zahra Rezaei Khavas, S Reza Ahmadzadeh, and Paul Robinette. 2020. Modeling trust in human-robot interaction: A survey. In International Conference on Social Robotics. 529-541.

[23] Bing Cai Kok and Harold Soh. 2020. Trust in robots: Challenges and opportunities. Current Robotics Reports (2020), 1-13.

[24] Allison Langer, Ronit Feingold-Polak, Oliver Mueller, Philipp Kellmeyer, and Shelly Levy-Tzedek. 2019. Trust in socially assistive robots: Considerations for use in rehabilitation. Neuroscience \& Biobehavioral Reviews (2019).

[25] Nicole Lazzeri, Daniele Mazzei, Lorenzo Cominelli, Antonio Cisternino, and Danilo Emilio De Rossi. 2018. Designing the mind of a social robot. Applied Sciences 8, 2 (2018), 302.

[26] Roger C Mayer, James H Davis, and F David Schoorman. 1995. An integrative model of organizational trust. Academy of management review 20, 3 (1995), 709-734.

[27] James C McCroskey and Thomas J Young. 1981. Ethos and credibility: The construct and its measurement after three decades. Communication Studies 32, 1 (1981), 24-34

[28] Wenxuan Mou, Martina Ruocco, Debora Zanatto, and Angelo Cangelosi. 2020. When Would You Trust a Robot? A Study on Trust and Theory of Mind in Human-Robot Interactions. In IEEE International Conference on Robot and Human Interactive Communication (RO-MAN). 956-962.

[29] PL Patrick Rau, Ye Li, and Dingjun Li. 2009. Effects of communication style and culture on ability to accept recommendations from robots. Computers in Human Behavior 25, 2 (2009), 587-595.

[30] Stephen Reysen. 2005. Construction of a new scale: The Reysen likability scale. Social Behavior and Personality: an international journal 33, 2 (2005), 201-208. 
[31] Maha Salem, Gabriella Lakatos, Farshid Amirabdollahian, and Kerstin Dautenhahn. 2015. Would you trust a (faulty) robot? Effects of error, task type and personality on human-robot cooperation and trust. In ACM/IEEE International Conference on Human-Robot Interaction (HRI). 1-8.

[32] Tracy Sanders, Alexandra Kaplan, Ryan Koch, Michael Schwartz, and Peter A Hancock. 2019. The relationship between trust and use choice in human-robot interaction. Human factors 61, 4 (2019), 614-626.

[33] Brian Scassellati. 2002. Theory of mind for a humanoid robot. Autonomous Robots 12, 1 (2002), 13-24.

[34] Eric Schniter, Timothy W Shields, and Daniel Sznycer. 2020. Trust in humans and robots: Economically similar but emotionally different. Journal of Economic Psychology 78 (2020), 102253.

[35] Pan Shu, Chen Min, Indu Bodala, Stefanos Nikolaidis, David Hsu, and Harold Soh. 2018. Human trust in robot capabilities across tasks. In Companion of ACM/IEEE International Conference on Human-Robot Interaction. 241-242.

[36] Stephanie Sturgeon, Andrew Palmer, Janelle Blankenburg, and David Feil-Seifer. 2019. Perception of Social Intelligence in Robots Performing False-Belief Tasks. In IEEE International Conference on Robot and Human Interactive Communication (RO-MAN). 1-7.

[37] Kimberly E Vanderbilt, David Liu, and Gail D Heyman. 2011. The development of distrust. Child development 82, 5 (2011), 1372-1380.

[38] Felix Warneken and Michael Tomasello. 2006. Altruistic helping in human infants and young chimpanzees. science 311, 5765 (2006), 1301-1303.

[39] Henry M Wellman and David Liu. 2004. Scaling of theory-of-mind tasks. Child development 75, 2 (2004), 523-541.

[40] Debora Zanatto. 2019. PhD Thesis: When do we cooperate with Robots? Investigations in Human-Robot Interaction and Trust. University of Plymouth (2019).

[41] Debora Zanatto, Massimiliano Patacchiola, Jeremy Goslin, and Angelo Cangelosi. 2019. Investigating cooperation with robotic peers. PloS one 14, 11 (2019)

[42] Debora Zanatto, Massimiliano Patacchiola, Jeremy Goslin, Serge Thill, and Angelo Cangelosi. 2020. Do humans imitate robots? An investigation of strategic social learning in human-robot interaction. In Proceedings of the ACM/IEEE International Conference on Human-Robot Interaction (HRI). 449-457. 